\title{
An Optimized Double Ramp Integrated Launch Package Design for Railguns
}

\author{
Jon R. KITZMILLER and Mircea D. DRIGA
}

\begin{abstract}
The need to evolve and make more efficient design tools for use in complex railgun system trade studies has led to an enhanced design for a fully integrated hybrid metal contact launch package for railguns. During work on two successful railgun projects at The University of Texas at Austin Center for Electromechanics (UT-CEM), an algorithm was designed which has produced what appears to be a strong design candidate for an aluminum double ramp integrated launch package (ILP). Using only four user inputs, the code produces railgun bore configurations (simple or augmented) and detailed ILPs which minimize the energy requirements for the compulsator. The output design gives useful mass fractions very close to the pinnacle of what is possible without more extensive use of composites.

This paper describes the design process used to optimize the railgun bore and ILP. Included is an advanced concept generated by the algorithm along with a detailed $3 D$ structural analysis of the ILP based upon a current profile that is relevant for high performance applications.
\end{abstract}

\section{INTRODUCTION}

$\mathrm{T}$ HE U.S. Army is interested in the development of electric armaments for the future combat field. A key component in this is the kinetic energy weapon featuring a long rod penetrator used to defeat main armor. The University of Texas at Austin Center for Electromechanics (UT-CEM) has performed research in this field for more than ten years, notably in two programs sponsored by the U.S. Army: Cannon Caliber Electromagnetic Gun (CCEMG), and Sabot Launched Electric Gun Kinetic Energy (SLEKE). In this research, UTCEM has taken the unique approach of studying and developing complete, detailed systems including integrated launch package (ILP), railgun, autoloader, switching, and pulse power supply. This system-wide experience has revealed that the design needs of advanced electric gun system components need to be addressed simultaneously in order to achieve viable system solutions.

A comprehensive design code is under development to

Manuscript received January 4, 2002. A portion of this research was carried out under the Electric Gun Program sponsored by the U.S. Army Research Laboratory through Lockheed-Martin Missile and Fire Control under contract number 4300050944.

J.R. Kitzmiller is affiliated with The University of Texas at Austin Center for Electromechanics, Austin, Texas 78758, telephone (512) 471-4496, fax (512) 471-0781.

M.D. Driga is affiliated with The University of Texas at Austin Department of Electrical and Computer Engineering, Austin, Texas 78712, telephone (512) 471-6943, fax (512) 471-3652. update and facilitate the "front end" design process (baseline details to meet a given set of performance goals) for use in advanced, alternator-driven railgun system trade studies. Early front end compulsator and switching designs had little or no coupling with the launcher and ILP. The most successful approach to designing these systems, however, seems to be that which starts with as much information as possible with the ILP including sub projectile performance on the target [1,2]. Generally, little is known about performance at the target using an innovative penetrator so it has been widely acceptable to use the total ILP muzzle kinetic energy to begin the design process. Given just this parameter along with the desired muzzle velocity, gun length, and number of shots desired to be stored inertially (mechanical and thermal), the design tool under development will produce a complete front end design of all system components. The first component to be addressed in code development is the ILP, the focus of this paper.

\section{ILP DESIGN METHODOLOGY}

UT-CEM was testing the first U.S. Army prototype iron core compulsator (CPA) when dramatic performance increases appeared as a result of moving from plasma to solidhybrid armatures [3]. The benefit in efficiency, particularly at lower velocities (under $4 \mathrm{~km} / \mathrm{s}$ ) had also been studied and verified by others including Barber [4]. For this application, the higher efficiency of the solid armature meant the CPA could operate at significantly less voltage and energy (reduced rotor speed). UT-CEM has since developed and tested a number of designs for solid armatures, including designs that are configured to carry long rod penetrators (Fig. 1).

The design code currently in use at UT-CEM features a balanced compilation of the most successful features of previous designs, along with a few new features designed to enhance the stability of the evolved design. The code uses a naturally converging algorithm that designs the advanced ILP completely, including the simultaneous design of the railgun geometry and approximate electrical current required to provide the forces to accelerate the projectile to target velocities. An experienced electric gun designer can accomplish this complex task by entering only a few values within two fundamental input categories.

- material properties - these inputs include the physical, mechanical, electrical, and thermal properties of the rails and armature/sabot components, along with the density 
and ultimate tensile yield strength of the penetrators; these inputs are typical for these systems and reside internal to the algorithm

- performance goals - these include items of interest to the end user such as muzzle kinetic energy and velocity, number of shots stored in the rails owning to thermal inertia considerations, acceleration length of the barrel, and acceleration and velocity ratio of the driving force applied to the ILP

Initialized with this information, the design algorithm then solves for valid ILP designs based upon variable useful mass fractions of the rod with respect to the total ILP. In order to produce credible subprojectile point designs, the rod diameter is scaled according to common practices considering existing cubed root rules for conventional munitions [5]. Given the mass fraction and rod diameter, the length is computed. From here the code solves viable designs for rod length to diameter ratios between 20 and 35 .

After the rod geometry has been defined, the armature is sized and initial armature mass and geometry developed, along with computed resistance and inductance gradients of a rail package supporting that geometry. Actions and accelerations are computed and critical geometry features of the armature/sabot are recomputed and a new calculation made of armature mass. This revised figure is compared to the earlier one and, if comparison parameters are not met, the process restarts with the revised design. This loop within the algorithm repeats until a converged design is found, if one is possible. The "best" design generally corresponds to the highest rod mass fraction of the ILP.

\section{MODEL DEVELOPMENT}

The armature/sabot model adopted for use builds from the previous work of Zielinski [6] and Price[1]. Fig. 2 shows the basic geometry upon which the model is built. The front and rear ramps are strain-matched to the penetrator through an exponential profile described using the penetrator and sabot mass densities, ILP peak acceleration, and yield stress allowable for the sabot:

$$
y_{\text {ramp }}=[R] \frac{1}{2}\left[\frac{\rho_{\text {rod }} A_{\text {rod }}}{\rho_{\text {sabot }}}\left(e^{\left(\frac{a_{\text {peak }} \rho_{\text {sabot }}}{\sigma_{\text {allowsabot }}}\right)\left(\frac{z_{\text {rod }}}{2}-z_{0}\right)}-1\right)\right]^{\frac{1}{2}}
$$

where

$$
\begin{aligned}
& x_{\text {ramp }}=\frac{1}{R} y_{\text {ramp }} \\
& A_{\text {ramp }}=y_{\text {ramp }} x_{\text {ramp }} ; \text { cross section of ramp } \\
& w_{\text {bore }}=y_{\text {ramp }} ; \text { width of bore (insulator to insulator) } \\
& y_{\text {ramp }}=" y " \text { dimension of ramp } \\
& x_{\text {ramp }}=" x " \text { dimension of ramp } \\
& R A A R=\text { ramp area aspect ratio }=R
\end{aligned}
$$

Note that equation (1) is used only for stress-matching, for strain-matching, this equation must be satisfied:

$$
\left\{\sigma_{s}=\frac{E_{s}}{E_{\rho}} \sigma_{\rho}\right\}
$$

The ramp area aspect ratio is generally held at one, but can be varied to permit more rail-to-rail stiffness of the ramps if needed for in-bore dynamic stability. The fin and mid section of the design are borrowed and enhanced forms of the largely successful CCEMG design [1] (enhancements are discussed below). The fin rake angle is set to $25^{\circ}$ as a compromise to fin rail-to-rail stiffness and location of the mass center of the package with respect to the stabilizer (bore rider). The structural design of the ILP depends on the peak current and corresponding acceleration. These values are computed for a simple bore geometry as shown in Fig. 3, using a high frequency 2D finite filament algorithm adopted from Leuer's method [7]. This algorithm was selected due to its favorable agreement with 3D finite element analyses run at UT-CEM.

There are three notable design modifications incorporated in the new design to enhance its survivability and accuracy in bore. First, the armature is a more robust design than seen on most previous packages, owing to a commitment from the power supply to not allow the current to drop off more than $75 \%$ of the peak value through acceleration. This led to the second modification, incorporating the bending strength of the armature in order to properly size the dimensions z3 and z4 (Fig. 2). This was accomplished by comparing the $\mathrm{z} 4$ dimension based upon thermal considerations and bending strength allowable using the derived expression for bending stress:

$$
\sigma=\frac{M * C}{I}
$$

where

$\sigma=$ bending stress located at $c$ distance from centroid

$M=$ bending moment defined by $\quad M=R_{a} * l_{f}-w * \frac{l^{2} f}{2}$

$I=$ area moment $M$ as defined by :

where $R_{a}$ is the net applied load to the fin, $l_{f}$ is the fin length, and $w$ is the fin thickness (width of the railgun bore).

It is important that the design output account for the bending stress, as it has been a driver in many designs previously run as test cases. Indeed, it can further separate fin dimensional features between simple and augmented cases where the load is reduced four to six times in the latter case. Care must also be taken to assure that the net loads applied to the armature fins force it against the rails continuously through its acceleration profile to help manage bore dynamic loads and stability of the ILP. It was this consideration that led to the last innovative feature of the design, a bore stabilizer developed (at some expense to maximum achievable mass fractions) as an exceptionally robust bore rider.

It is critical in the design of electric guns that the firing signature be minimized by removing (or, at least, drastically diminishing) the current in the ILP armature before it leaves the muzzle of the launcher. If the armature is forced to switch off the power supply current by its natural exit from the barrel, 
large tip off forces, significant barrel damage, and significant barrel signatures can occur simultaneously. Unfortunately, removal of the current from the armature in-bore has the potential to separate the ablated hybrid armature contacts from the rails. If the armatures were intended to provide in-bore structural support or centering of the ILP, the sudden disruption of this function can produce catastrophic negative effects, such as failure within the ILP and/or wide dispersion in accuracy and unmanageable rod angular rates in flight.

The newly designed bore stabilizer provides centering and support for the ILP, after current is removed from the armature prior to muzzle exit, and is effective regardless of the in-bore condition of the armature. This feature should provide a good measure of support and enhance subprojectile survivability (from tips and tails breaking off) as well as improved accuracy.

\section{Model Example Design and Preliminary VERIFICATION}

The model for this design was assembled and executed in FORTRAN and results were compared to many earlier designs [1-11]. For the purpose of validation, an ambiguous 2,300 m/s launch package was designed and validated using a dynamic 3D mechanical finite element analysis. It must be noted that a presumed current path was used to apply the time marching loads within the ILP; further analyses would better rely on 3D current distribution from an accredited electromagnetic code. Pertinent output from the design code is listed in table 1. For this case, the code designs a $40 \%$ useful mass fraction ILP into a rectangular gun bore $64 \mathrm{~mm} \times 133 \mathrm{~mm}$ or $105 \mathrm{~mm}$ equivalent round bore area.

Structural verification was carried out using COSMOS/M structural analysis software. The model was first developed within COSMOS/M and is shown in Fig. 4 (as the design is symmetric, only a quarter section was modeled). The magnetic force was calculated and applied throughout the back third region of the armature and fin based upon circuit simulation from the power supply output matching this design. The force profile used in this case is shown in Fig. 5. Notice the armature current is forced to zero; this is while it is still inbore and is representative of current transferring to a passive muzzle shunt, which is also modeled in the circuit simulation. Again, it is important to point out that the proposed stabilizer maintains structural support and centering while the ILP is inbore. Figs. 6 and 7 show the equivalent maximum stress contours in the armature/sabot which occur just after peak current in the dynamic analysis. Finally, as proof the load is representative, Fig. 8 shows the ILP velocity profile which confirms that the target of $2,300 \mathrm{~m} / \mathrm{s}$ was attained for assumed force distribution.

\section{CONCLUSIONS AND RECOMMENDATIONS}

The development of an efficient frontal electric gun system design code has resulted in the concept design of an advanced ILP that would seem to hold great promise of producing a successful integrated launch package. The design encompasses many working features of previous designs along with some new features which should increase its general performance. This is especially true for gun/power supply designs which attempt to remove the current from the armature prior to muzzle exit.

Preliminary analyses have shown the design to be viable. Prior to railgun testing, additional detailed electromagnetic and barrel dynamic analyses will be required to ensure proper performance.

\section{ACKNOWLEDGMENT}

The authors wish to express their appreciation to Alex Zielinski of the U.S. Army Research Laboratory, for his insight and previous hard work.

\section{REFERENCES}

[1] J.H. Price, et al, "Discarding Armature and Barrel Optimization for a Cannon Caliber Electromagnetic Launch System," 7Th EML April 2024 1994, IEEE Transmag Vol 31, No 1, January 1995.

[2] A.E. Zielinski, "A Projectile Oriented Design Study for a CannonCaliber Electromagnetic Launcher," 6Th EML April 21-24 1992, IEEE Transmag Vol 29, No 1, January 1993, Pgs 889-893.

[3] M.D. Werst, D.E. Perkins, et al., "Testing of a Rapid Fire Compensated Pulsed Alternator System," 4Th EML, IEEE TransMag Jan 1989 Vol 25, Pg 599-604.

[4] J.H. Price, et al. "Results of Monolithic Solid Armature Tests in a Railgun," 1987 International Current Collector Conference, Nov 16-17.

[5] A.E. Zielinski, "End State Integrated Launch Package Design as a Basis for Assessing Augmentation," IAT Internal Technical Report TN 0180, March 2000.

[6] A.E. Zielinski, " Saboted Rod Projectile for Electromagnetic Launch," Proceeding from 12th International Symposium on Ballistics, San Antonio, TX, October 1990.

[7] J.A. Leuer, "Electromagnetic Modeling of Complex Railgun Geometries," IEEE Transmag, Vol 22, No 6, pp 1584-1590, November 1986.

[8] J.H. Price, et al, "Design and Testing of Solid Armatures for Large-Bore Railguns," 4Th EML, 1989,IEEE TransMag Vol 25, Pg 467-474.

[9] J.H. Price and M.W. Ingram, "Large and Small Caliber Railgun Solid Armatures; Experimental Results," 5th EML April 1990, IEEE Transmag 1991 Vol 27 No. 1.

[10] J.H. Price and H.D. Yun, "Design and Testing of Integrated Metal Armature Sabots for Launch of Armor Penetrating Projectiles from Electric Guns," 7Th EML April 20-24 1994, IEEE Transmag Vol 31, No 1, January 1995.

[11] R.C. Zowarka, H.D. Yun, "Railgun Solid Armature Scaling Model," 8th EML April 21-24 1996, IEEE Transmag Vol 33, No 1, January 1997, Pgs 169-174. 
Table 1: Design Output for Test Case

\begin{tabular}{lcc}
\hline \hline \multicolumn{1}{c}{ Parameter } & Units & Value \\
\hline Muzzle velocity & $\mathrm{m} / \mathrm{s}$ & 2,300 \\
Peak current & $\mathrm{MA}$ & 3.375 \\
Peak acceleration & $\mathrm{kgees}$ & 80 \\
Gun length (acceleration) & $\mathrm{m}$ & 5 \\
Useful mass fraction & $\%$ & $>35$ \\
Rod diameter & $\mathrm{mm}$ & 16 \\
Rod L/D & - & $>15$ \\
Gun L' & $\mathrm{H} / \mathrm{m}$ & $0.58 \times 10^{-6}$ \\
Gun R' & $\Omega / \mathrm{m}$ & $48 \times 10^{-6}$ \\
Gun bore width & $\mathrm{mm}$ & 64 \\
Gun bore Height & $\mathrm{mm}$ & 133 \\
Equivalent round bore & $\mathrm{mm}$ & 105 \\
\hline \hline
\end{tabular}
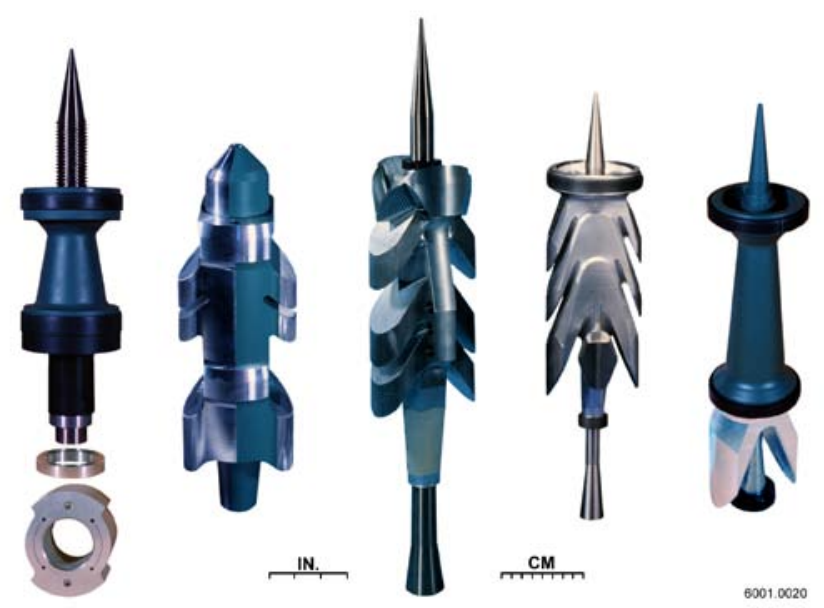

Figure 1. Electric gun ILPs developed at UT-CEM

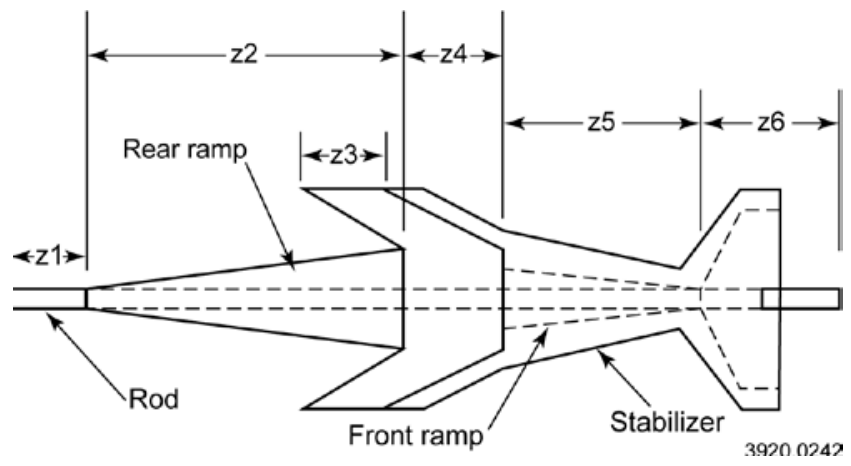

Figure 2. ILP longitudinal model used in design algorithm

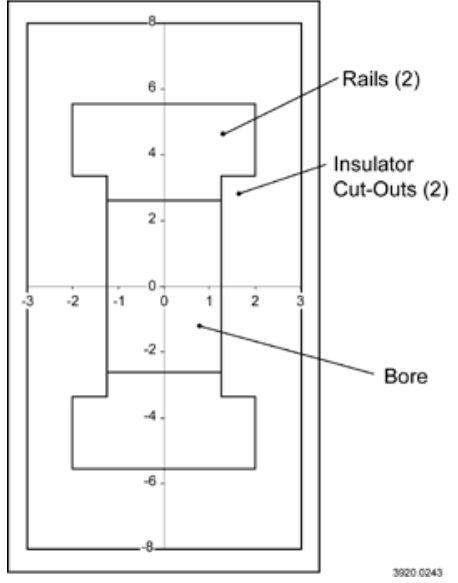

Figure 3. Railgun bore cross section

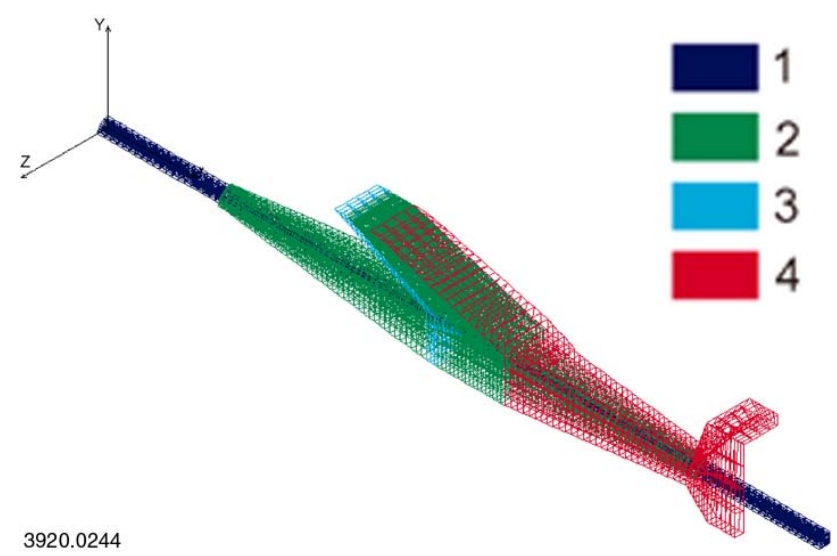

Figure 4. 3D FEA of ILP for verification

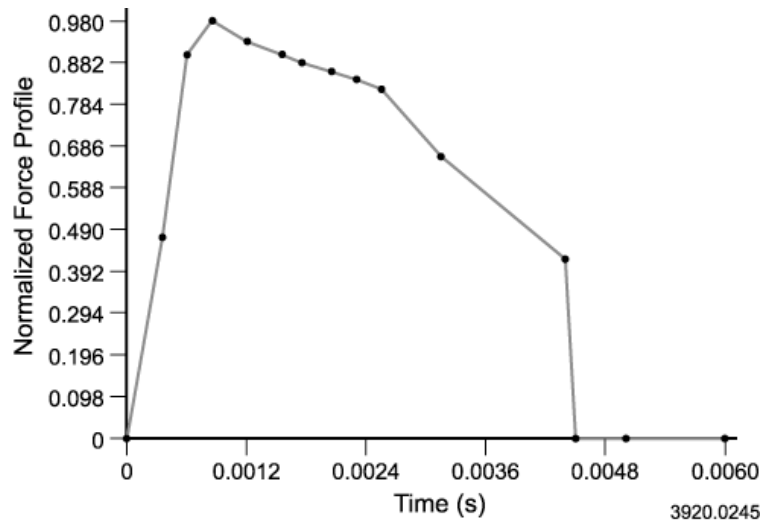

Figure 5. Normalized force profile for model input 


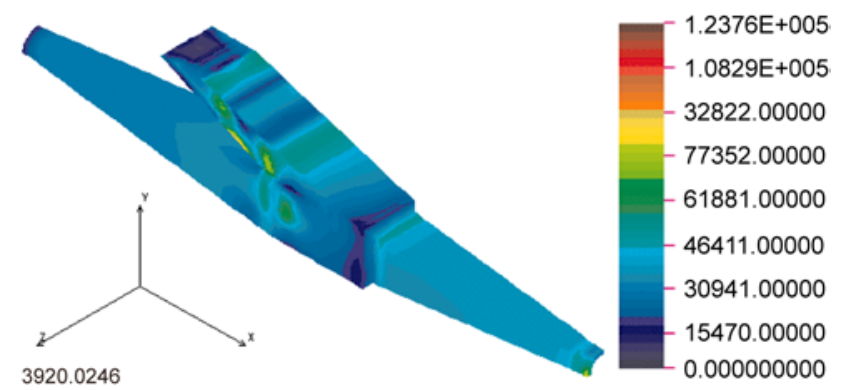

Figure 6. Von Mises stress contours for armature/sabot

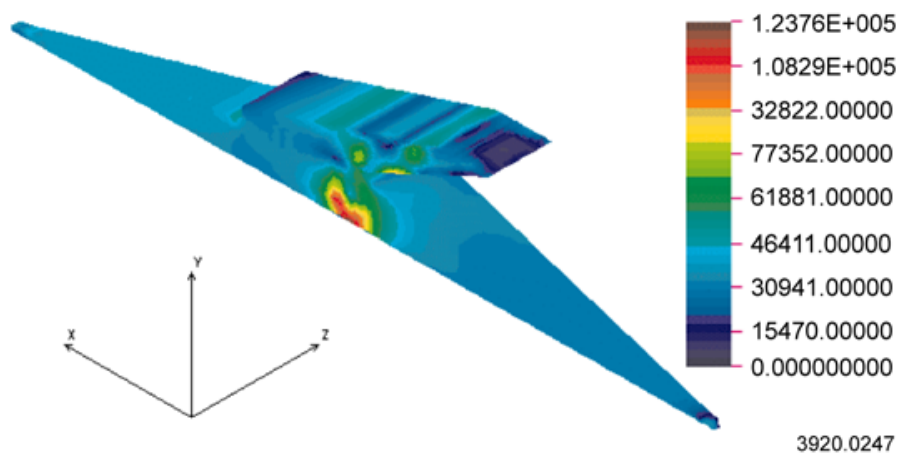

Figure 7. Von Mises stress contours for armature/sabot (reverse angle)

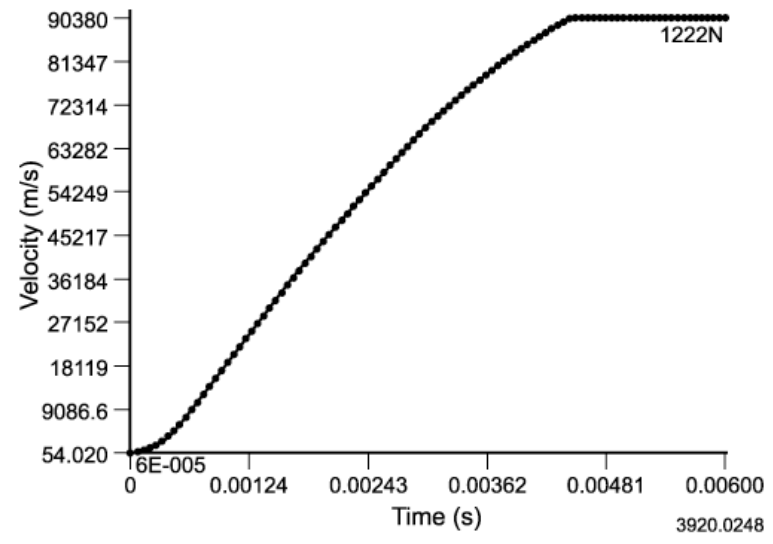

Figure 8. Velocity vs. time for applied load in structural analysis 